\title{
Norms on Direct Sums and Tensor Products
}

\author{
By P. Lancaster and H. K. Farahat
}

\begin{abstract}
We first consider the construction of a norm on a direct sum of normed linear spaces and call a norm absolute if it depends only on the norms of the component spaces. Several characterizations are given of absolute norms. Absolute norms are then used to construct norms.on tensor products of normed linear spaces and on tensor products of operators on normed linear spaces.
\end{abstract}

1. Introduction. In this paper, we consider the construction of norms on composite linear spaces formed from direct sums and tensor products of normed linear spaces and we consider properties of norms of operators on these spaces. The notion of an absolute norm is introduced as a natural generalization of the relatively familiar idea of an absolute vector norm on the space $C_{n}$ of ordered $n$-tuples of complex numbers. Such norms on $C_{n}$ correspond to the "coordinatewise symmetric" gauge functions as described by Ostrowski [3], and it is shown that our absolute norms on composite spaces correspond in a one-to-one fashion with the absolute vector norms on $C_{n}$.

We are particularly interested in operator norms for which, in an appropriate sense to be detailed later,

$$
\|A \otimes B\|=\|A\|\|B\|
$$

where $A, B$ are linear operators on linear spaces and $\otimes$ denotes the tensor product of linear operators.

In Sections 2 and 3, we introduce absolute norms on direct sums of normed linear spaces and obtain several characterizations of them. In Section 4, we discuss norms on tensor products of linear spaces and exploit the "absolute" norm idea. In essence, we are looking for a definition of a "natural" norm in a space $L$ which is the tensor product of normed linear spaces $X$ and $Y$. One desirable property is that the operator norms induced from those on $X, Y$ and $L$ should have the property displayed above which defines a crossnorm (for a vector or operator norm). In Section 4, we make connections between absolute norms and crossnorms.

Norms of tensor products of operators are discussed in Section 5 and, in Section 6, we illustrate our results with applications to complex matrices.

2. Absolute Norms. In this paper, all linear spaces are over the complex numbers $C$. We frequently need to consider the supremum of sets of real numbers formed from quotients. In such cases, it is tacitly assumed that the supremum is restricted to a set for which the denominator is nonzero.

Let $X_{1}, X_{2}, \cdots, X_{n}$ be normed linear spaces and let $X$ denote the direct sum $X=X_{1} \oplus X_{2} \oplus \cdots \oplus X_{n}=\sum_{i} \oplus X_{i}$. If $x, y \in X$ and $x=\left(x_{1}, x_{2}, \cdots, x_{n}\right)$,

Received August 9, 1971.

AMS 1970 subject classifications. Primary 46B99, 65F35, 15A60.

Copyright (c) 1972, American Mathematical Society 
$y=\left(y_{1}, y_{2}, \cdots, y_{n}\right)$, we say that a norm on $X$ is absolute if $\left\|x_{i}\right\|=\left\|y_{i}\right\|$, $j=1,2, \cdots, n$, implies $\|x\|=\|y\|$.

A norm on $X$ is monotonic if $\left\|x_{i}\right\| \leqq\left\|y_{i}\right\|, j=1,2, \cdots, n$, implies $\|x\| \leqq\|y\|$.

Our first results generalize theorems of Bauer, Stoer, and Witzgall [1], which may be interpreted as the special cases of our results in which the given normed linear spaces are one-dimensional.

THEOREM 1. A norm on $X$ is absolute iff it is monotonic.

Proof. Suppose first that the norm is monotonic. Then $\left\|x_{i}\right\|=\left\|y_{i}\right\|$, $j=1,2, \cdots, n$, implies $\|x\| \leqq\|y\|$ and $\|y\| \leqq\|x\|$, whence $\|x\|=\|y\|$ and the norm is absolute.

Conversely, if the norm is absolute, we have

$$
\begin{aligned}
\left\|\left(0, x_{2}, \cdots, x_{n}\right)\right\| & =\left\|\frac{1}{2}\left(x_{1}, x_{2}, \cdots, x_{n}\right)+\frac{1}{2}\left(-x_{1}, x_{2}, \cdots, x_{n}\right)\right\| \\
& \leqq \frac{1}{2}\left\|\left(x_{1}, \cdots, x_{n}\right)\right\|+\frac{1}{2}\left\|\left(-x_{1}, x_{2}, \cdots, x_{n}\right)\right\| \\
& =\left\|\left(x_{1}, x_{2}, \cdots, x_{n}\right)\right\| .
\end{aligned}
$$

Now, suppose that $\lambda=\left\|x_{1}\right\| /\left\|x_{1}^{\prime}\right\| \leqq 1$. Then,

$$
\begin{aligned}
\left\|\left(x_{1}, x_{2}, \cdots, x_{n}\right)\right\| & =\left\|\left(\lambda x_{1}^{\prime}, x_{2}, \cdots, x_{n}\right)\right\| \\
& =\left\|\lambda\left(x_{1}^{\prime}, x_{2}, \cdots, x_{n}\right)+(1-\lambda)\left(0, x_{2}, \cdots, x_{n}\right)\right\| \\
& \leqq \lambda\left\|\left(x_{1}^{\prime}, x_{2}, \cdots, x_{n}\right)\right\|+(1-\lambda)\left\|\left(0, x_{2}, \cdots, x_{n}\right)\right\| \\
& \leqq \lambda\left\|\left(x_{1}^{\prime}, x_{2}, \cdots, x_{n}\right)\right\|+(1-\lambda)\left\|\left(x_{1}^{\prime}, x_{2}, \cdots, x_{n}\right)\right\|
\end{aligned}
$$

using inequality (1). It follows that $\left\|x_{1}\right\| \leqq\left\|x_{1}^{\prime}\right\|$ implies

$$
\left\|\left(x_{1}, x_{2}, \cdots, x_{n}\right)\right\| \leqq\left\|\left(x_{1}^{\prime}, x_{2}, \cdots, x_{n}\right)\right\| .
$$

Since a similar argument applies to each position of $x$, it follows that the norm on $X$ is monotonic.

Suppose that, for $j=1,2, \cdots, n, A_{i}$ is a linear operator on $X_{i}$. Then, a linear operator $A=A_{1} \oplus \cdots \oplus A_{n}$ on $X=\sum_{i} \oplus X_{i}$ is defined by

$$
\left(A_{1} \oplus A_{2} \oplus \cdots \oplus A_{n}\right)\left(x_{1}, x_{2}, \cdots, x_{n}\right)=\left(A_{1} x_{1}, A_{2} x_{2}, \cdots, A_{n} x_{n}\right)
$$

for all $x_{i} \in X_{i}, j=1,2, \cdots, n$. We consider now the so-called "bound" norm for operators; that is, the norm induced by the norm of the space on which the operator acts. Thus, $\|A\|=\sup \|A x\| /\|x\|$.

With the above notations, we say that a norm on $X=\sum_{i} \oplus X_{i}$ has the maximum property if, for any linear operator $A=\sum_{i} \oplus A_{i}$, we have $\|A\|=\max _{i}\left\|A_{i}\right\|$. We are going to show that the norm on $X$ is absolute iff the induced operator norm has the maximum property.

Lemma. Let $X$ be a normed linear space and $x, x^{\prime} \in X$ with $\|x\|=\left\|x^{\prime}\right\|$. Then, there exists a linear operator $A$ on $X$ such that $A x=x^{\prime}$ and $\|A\|=1$.

Proof. Let $X^{*}$ be the space of continuous linear functionals on $X$ with the usual norm. We shall call $X^{*}$ the dual of $X$. It is well-known (Dunford and Schwartz [2] II.3.14) that, given a nonzero $x \in X$, there is an $x^{*} \in X^{*}$ for which $x^{*} x=\|x\|$ and $\left\|x^{*}\right\|=1$. We define the linear operator $A$ on $X$ by

$$
A y=\left(x^{*}(y) /\|x\|\right) x^{\prime}
$$


for all $y \in X$. Then, we obviously have $A x=x^{\prime}$ and, since $\|x\|=\left\|x^{\prime}\right\|$,

$$
\|A\|=\sup _{\nu \in X} \frac{\|A y\|}{\|y\|}=\sup _{\nu} \frac{\left\|x^{*}(y) x^{\prime}\right\|}{\|x\|\|y\|}=\sup _{\nu} \frac{\left|x^{*}(y)\right|}{\|y\|}=\left\|x^{*}\right\|=1 .
$$

In the case $\|x\|=\left\|x^{\prime}\right\|=0$, we simply choose $A=I$.

THEOREM 2. A norm on $X$ is absolute iff it has the maximum property.

Proof. Suppose first that the norm on $X$ has the maximum property and let $x, y \in X$ with $\left\|x_{j}\right\|=\left\|y_{j}\right\|, j=1,2, \cdots, n$. We are to prove $\|x\|=\|y\|$. By the lemma, there exist linear operators $A_{i}$ on $X_{i}$ such that $A_{i} x_{i}=y_{i}$ and $\left\|A_{i}\right\|=1$ for each $j$. Then, using the maximum property,

$$
\begin{aligned}
\left\|\left(y_{1}, y_{2}, \cdots, y_{n}\right)\right\| & =\left\|\left(A_{1} x_{1}, A_{2} x_{2}, \cdots, A_{n} x_{n}\right)\right\| \\
& =\left\|\left(A_{1} \oplus \cdots \oplus A_{n}\right)\left(x_{1}, x_{2}, \cdots, x_{n}\right)\right\| \\
& \leqq\left\|\left(x_{1}, x_{2}, \cdots, x_{n}\right)\right\| \max _{i}\left\|A_{i}\right\|=\left\|\left(x_{1}, x_{2}, \cdots, x_{n}\right)\right\| .
\end{aligned}
$$

Thus, $\|y\| \leqq\|x\|$. However, reversing the roles of $x$ and $y$ we can also prove $\|x\| \leqq\|y\|$ and, hence, $\|x\|=\|y\|$.

Conversely, suppose it given that the norm on $X$ is absolute and let $A=\sum_{i} \oplus A_{i}$ be a direct sum of linear operators on the spaces $X_{i}$. If $\mu=\max _{i}\left\|A_{i}\right\|$, we are to prove that $\|A\|=\mu$. Now, for each $j=1,2, \cdots, n$,

$$
\left\|A_{j} x_{i}\right\| \leqq\left\|A_{i}\right\|\left\|x_{i}\right\| \leqq \mu\left\|x_{i}\right\|=\left\|\mu x_{i}\right\|
$$

and, since an absolute norm is monotonic,

$$
\|A x\|=\left\|\left(A_{1} x_{1}, \cdots, A_{n} x_{n}\right)\right\| \leqq\left\|\left(\mu x_{1}, \cdots, \mu x_{n}\right)\right\|=\mu\|x\| .
$$

It follows immediately that $\|A\| \leqq \mu$.

To prove the reverse inequality, we have, for $x_{1} \in X_{1}, x_{1} \neq 0$,

$$
\|A\| \geqq \frac{\left\|A\left(x_{1}, 0, \cdots, 0\right)\right\|}{\left\|\left(x_{1}, 0, \cdots, 0\right)\right\|}=\frac{\left\|\left(A_{1} x_{1}, 0, \cdots, 0\right)\right\|}{\left\|\left(x_{1}, 0, \cdots, 0\right)\right\|} .
$$

If $\mu\left(x_{1}\right)=\left\|A_{1} x_{1}\right\| /\left\|x_{1}\right\|$, then $\left\|A_{1} x_{1}\right\|=\left\|\mu\left(x_{1}\right) x_{1}\right\|$ and, since the norm is absolute, $\|A\| \geqq\left\|\left(\mu\left(x_{1}\right) x_{1}, 0, \cdots, 0\right)\right\| /\left\|\left(x_{1}, 0, \cdots, 0\right)\right\|=\mu\left(x_{1}\right)$.

Taking the supremum over all nonzero $x_{1} \in X_{1}$, we have $\|A\| \geqq\left\|A_{1}\right\|$. In a similar way, we obtain $\|A\| \geqq\left\|A_{i}\right\|, j=1,2, \cdots, n$, and we have $\|A\| \geqq \mu$.

3. Connection with Norms on $C_{n}$. If $C$ denotes the linear space of the complex numbers with the absolute value norm, we write $C_{n}=C \oplus C \oplus \cdots \oplus C$ ( $n$ times). Then, a norm on $C_{n}$ is absolute iff it is a function of the absolute values of the components of the members of $C_{n}$. Suppose once more that $X_{1}, \cdots, X_{n}$ are normed linear spaces and that we have an absolute norm on $X=\sum_{i} \oplus X_{i}$. Define a function $h$ on $C_{n}$ by

$$
h\left(\xi_{1}, \xi_{2}, \cdots, \xi_{n}\right)=\left\|\left(x_{1}, x_{2}, \cdots, x_{n}\right)\right\|,
$$

where $\left(\xi_{1}, \cdots, \xi_{n}\right) \in C_{n}, x_{i} \in X_{i}$, and $\left\|x_{i}\right\|=\left|\xi_{i}\right|, j=1,2, \cdots, n$. If $u_{i} \in X_{i}$ and $\left\|u_{i}\right\|=1$, then $\left\|\xi_{i} u_{i}\right\|=\left|\xi_{i}\right|$ and we may write

$$
h\left(\xi_{1}, \xi_{2}, \cdots, \xi_{n}\right)=\left\|\left(\xi_{1} u_{1}, \xi_{2} u_{2}, \cdots, \xi_{n} u_{n}\right)\right\| .
$$


THEOREM 3. (a) Every absolute norm $\|\cdot\|$ on $X$ defines, by (2), an absolute norm $h$ on $C_{n}$. (b) Conversely, every absolute norm $h$ on $C_{n}$ defines, by (2) again, an absolute norm $\|\cdot\|$ on $X$.

Proof. (a) Using formula (3), we have

$$
h\left(\xi_{1}, \xi_{2}, \cdots, \xi_{n}\right)=0 \Rightarrow\left\|\left(\xi_{1} u_{1}, \cdots, \xi_{n} u_{n}\right)\right\|=0 \Rightarrow \xi_{j} u_{i}=0 \Rightarrow \xi_{i}=0
$$

for $j=1,2, \cdots, n$.

$$
\text { (ii) } \begin{aligned}
h\left(\lambda \xi_{1}, \lambda \xi_{2}, \cdots, \lambda \xi_{n}\right)= & \left\|\left(\lambda \xi_{1} u_{1}, \cdots, \lambda \xi_{n} u_{n}\right)\right\| \\
= & |\lambda|\left\|\left(\xi_{1} u_{1}, \cdots, \xi_{n} u_{n}\right)\right\|=|\lambda| h\left(\xi_{1}, \xi_{2}, \cdots, \xi_{n}\right) . \\
h\left(\left(\xi_{1}, \cdots, \xi_{n}\right)+\left(\eta_{1}, \cdots, \eta_{n}\right)\right) & =h\left(\xi_{1}+\eta_{1}, \cdots, \xi_{n}+\eta_{n}\right) \\
& =\left\|\left(\left(\xi_{1}+\eta_{1}\right) u_{1}, \cdots,\left(\xi_{n}+\eta_{n}\right) u_{n}\right)\right\| \\
& =\left\|\left(\xi_{1} u_{1}, \cdots, \xi_{n} u_{n}\right)+\left(\eta_{1} u_{1}, \cdots, \eta_{n} u_{n}\right)\right\| \\
& \leqq \\
& \leqq \\
& =h\left(\xi_{1} u_{1}, \cdots, \xi_{n} u_{n}\right)\|+\|\left(\eta_{1} u_{1}, \cdots, \eta_{n} u_{n}\right) \|
\end{aligned}
$$

This shows that $h$ is a norm on $C_{n}$ and it is clearly absolute.

(b) Now, suppose it given that $h$ is an absolute norm on $C_{n}$ and define a function $\|\cdot\|$ on $X$ by means of (2). Then, if $\left\|u_{i}\right\|=1$, for $j=1,2, \cdots, n$, it follows that

$$
\left\|\left(x_{1}, \cdots, x_{n}\right)\right\|=\left\|\left(\xi_{1} u_{1}, \cdots, \xi_{n} u_{n}\right)\right\|
$$

where $\left|\xi_{i}\right|=\left\|x_{i}\right\|, j=1,2, \cdots, n$. Then, we have

$$
\begin{aligned}
& \left\|\left(x_{1}, \cdots, x_{n}\right)\right\|=0 \Rightarrow h\left(\xi_{1}, \cdots, \xi_{n}\right)=0 \text { and }\left|\xi_{i}\right|=\left\|x_{i}\right\| \\
& \Rightarrow \xi_{i}=0 \Rightarrow x_{i}=0 . \\
& \left\|\lambda\left(x_{1}, \cdots, x_{n}\right)\right\|=\left\|\left(\lambda x_{1}, \cdots, \lambda x_{n}\right)\right\|=h\left(|\lambda| \xi_{1}, \cdots,|\lambda| \xi_{n}\right) \\
& =|\lambda| h\left(\xi_{1}, \cdots, \xi_{n}\right)=|\lambda|\left\|\left(x_{1}, \cdots, x_{n}\right)\right\| \text {. } \\
& \left\|\left(x_{1}, \cdots, x_{n}\right)+\left(y_{1}, \cdots, y_{n}\right)\right\|=\left\|\left(x_{1}+y_{1}, \cdots, x_{n}+y_{n}\right)\right\| \\
& =h\left(\left\|x_{1}+y_{1}\right\|, \cdots,\left\|x_{n}+y_{n}\right\|\right) \\
& \leqq h\left(\left\|x_{1}\right\|+\left\|y_{1}\right\|, \cdots,\left\|x_{n}\right\|+\left\|y_{n}\right\|\right),
\end{aligned}
$$

using the fact that an absolute norm is monotonic. Thus,

$$
\begin{aligned}
\left\|\left(x_{1}, \cdots, x_{n}\right)+\left(y_{1}, \cdots, y_{n}\right)\right\| & \leqq h\left(\left(\left\|x_{1}\right\|, \cdots,\left\|x_{n}\right\|\right)+\left(\left\|y_{1}\right\|, \cdots,\left\|y_{n}\right\|\right)\right) \\
& \leqq h\left(\left\|x_{1}\right\|, \cdots,\left\|x_{n}\right\|\right)+h\left(\left\|y_{1}\right\|, \cdots,\left\|y_{n}\right\|\right) \\
& =\left\|\left(x_{1}, \cdots, x_{n}\right)\right\|+\left\|\left(y_{1}, \cdots, y_{n}\right)\right\| .
\end{aligned}
$$

This completes the proof.

There is a close analogy between the result of this theorem and Theorem 5.2 of Schatten [4], in which he identifies unitarily invariant crossnorms on a Hilbert space with symmetric gauge functions. In our next result, we show that the correspondence obtained in Theorem 3 extends to the norms on the dual spaces of $X$ and $C_{n}$. First, we recall that, for any normed linear spaces $X_{1}, X_{2}, \cdots, X_{n}$, the algebraic isomorphism between 


$$
X^{*}=\left(X_{1} \oplus X_{2} \oplus \cdots \oplus X_{n}\right)^{*} \quad \text { and } \quad X_{1}^{*} \oplus X_{2}^{*} \oplus \cdots \oplus X_{n}^{*}
$$

allows us to identify these two spaces. Thus, if $z^{*} \in X^{*}$, we identify $z^{*}$ with $\left(x_{1}^{*}, \cdots, x_{n}^{*}\right)$ and we have $z^{*}\left(x_{1}, \cdots, x_{n}\right)=\sum_{i} x_{i}^{*} x_{i}$. We first need a lemma which parallels Theorem 1 of Bauer, Stoer and Witzgall [1].

Lemma. If $C_{n}$ has an absolute norm $h$, then the induced norm $h^{*}$ on $C_{n}^{*}$ is also absolute.

Proof. As above, we identify $C_{n}^{*}=(C \oplus \cdots \oplus C)^{*}$ with $C^{*} \oplus \cdots \oplus C^{*}$ and note that each element of $C^{*}$ is a multiplication by a complex number with the absolute value of this number as norm.

Let $\left(\xi_{1}^{*}, \cdots, \xi_{n}^{*}\right) \in C^{*} \oplus \cdots \oplus C^{*}$ and let $\xi_{i}^{*}$ be multiplication by the complex number $\alpha_{i} \exp \left(i \theta_{j}\right)$ where $\alpha_{i} \geqq 0$. Then, taking a supremum over the set $S$ of vectors $\left(\xi_{1}, \cdots, \xi_{n}\right) \in C_{n}$ with unit norm,

$$
\begin{aligned}
h^{*}\left(\xi_{1}^{*}, \cdots, \xi_{n}^{*}\right) & =\sup \left|\xi_{1}^{*}\left(\xi_{1}\right)+\cdots+\xi_{n}^{*}\left(\xi_{n}\right)\right| \\
& =\sup \left|\alpha_{1} \xi_{1} \exp \left(i \theta_{1}\right)+\cdots+\alpha_{n} \xi_{n} \exp \left(i \theta_{n}\right)\right| .
\end{aligned}
$$

Let $\eta_{i}=\xi_{i} \exp \left(i \theta_{i}\right)$ so that $h\left(\eta_{1}, \cdots, \eta_{n}\right)=h\left(\xi_{1}, \cdots, \xi_{n}\right)$ (since the norm on $C_{n}$ is absolute) and take the supremum now over vectors $\left(\eta_{1}, \cdots, \eta_{n}\right) \in S$ (obviously the whole of $S$ ):

$$
h^{*}\left(\xi_{1}^{*}, \cdots, \xi_{n}^{*}\right)=\sup \left|\alpha_{1} \eta_{1}+\cdots+\alpha_{n} \eta_{n}\right|=h^{*}\left(\tilde{\alpha}_{1}, \cdots, \tilde{\alpha}_{n}\right),
$$

where $\tilde{\alpha}_{i}$ is multiplication (of $C$ ) by $\alpha_{i}$. Thus,

$$
h^{*}\left(\xi_{1}^{*}, \cdots, \xi_{n}^{*}\right)=h^{*}\left(\left\|\xi_{1}^{*}\right\|, \cdots,\left\|\xi_{n}^{*}\right\|\right) .
$$

THEOREM 4. Given an absolute norm $\|\cdot\|$ on $X\left(=\sum_{i} \oplus X_{i}\right)$, let the associated norm on $C_{n}$ (as in the lemma) be $h$. Then, the norm on $X^{*}$ is absolute and is associated with the norm $h^{*}$ on $C_{n}^{*}$. That is, if $\left(x_{1}^{*}, \cdots, x_{n}^{*}\right) \in X^{*},\left\|\left(x_{1}^{*}, \cdots, x_{n}^{*}\right)\right\|=$ $h^{*}\left(\tau_{1}, \cdots, \tau_{n}\right)$, where $\tau_{j} \in C^{*}$ and $\left\|\tau_{i}\right\|=\left\|x_{i}^{*}\right\|$.

Proof. Let $u_{i} \in X_{i}$ with $\left\|u_{i}\right\|=1, j=1,2, \cdots, n$. These vectors determine a linear operator $\theta: C_{n} \rightarrow X$ by means of $\theta\left(\xi_{1}, \cdots, \xi_{n}\right)=\left(\xi_{1} u_{1}, \cdots, \xi_{n} u_{n}\right)$ which (by (3)) is norm-preserving. Hence, $\theta$ has unit norm. Now, the composition $\left(x_{1}^{*}, \cdots, x_{n}^{*}\right) \theta$ is a linear operator from $C_{n}$ to $C$, so we have

$$
h^{*}\left(\left(x_{1}^{*}, \cdots, x_{n}^{*}\right) \theta\right) \leqq\left\|\left(x_{1}^{*}, \cdots, x_{n}^{*}\right)\right\|\|\theta\|=\left\|\left(x_{1}^{*}, \cdots, x_{n}^{*}\right)\right\|
$$

and, taking the following supremum over elements $\left(\xi_{1}, \cdots, \xi_{n}\right) \in \dot{C_{n}}$ of unit $h$-norm,

$$
\begin{aligned}
h^{*}\left(\left(x_{1}^{*}, \cdots, x_{n}^{*}\right) \theta\right) & =\sup \left|\sum_{i} x_{i}^{*}\left(\xi_{i} u_{i}\right)\right|=\sup \left|\sum_{i} \xi_{i} x_{i}^{*}\left(u_{i}\right)\right| \\
& =h^{*}\left(x_{1}^{*}\left(u_{1}\right) \tilde{}, \cdots, x_{n}^{*}\left(u_{n}\right) \tilde{)}\right),
\end{aligned}
$$

where $x_{i}^{*}\left(u_{i}\right)^{\sim}$ is the linear functional corresponding to multiplication of $C$ by $x_{i}^{*}\left(u_{i}\right)$. Thus, from (4), we have

$$
\left\|\left(x_{1}^{*}, \cdots, x_{n}^{*}\right)\right\| \geqq h^{*}\left(x_{1}^{*}\left(u_{1}\right)^{\tilde{2}}, \cdots, x_{n}^{*}\left(u_{n}\right) \tilde{)}\right),
$$

whenever $\left\|u_{i}\right\|=1, j=1,2, \cdots, n$, and, hence,

$$
\left\|\left(x_{1}^{*}, \cdots, x_{n}^{*}\right)\right\| \geqq s,
$$

where $s=\sup _{\left\|u_{i}\right\|=1} h^{*}\left(\left|x_{1}^{*}\left(u_{1}\right)\right|^{\sim}, \cdots,\left|x_{n}^{*}\left(u_{n}\right)\right|^{\sim}\right)$ and we have used the fact that $h^{*}$ is absolute. It follows from the definition of $\left\|x_{i}^{*}\right\|$ that, given $\epsilon>0$, there exists 
(for each $j$ ) a $v_{i} \in X_{i}$ such that $\left\|v_{i}\right\|=1$ and $\left|x_{i}^{*}\left(v_{i}\right)\right|>\left\|x_{i}^{*}\right\|-\epsilon$. Using the monotonic property of $h^{*}$, we therefore have

$$
\begin{aligned}
& h^{*}\left(\left\|x_{1}^{*}\right\|^{\tilde{1}}, \cdots,\left\|x_{n}^{*}\right\|^{\tilde{})} \leqq h^{*}\left(\left(x_{1}^{*}\left(v_{1}\right)+\epsilon\right)^{\tilde{2}}, \cdots,\left(x_{n}^{*}\left(v_{n}\right)+\epsilon \tilde{)}\right)\right.\right. \\
& \leqq h^{*}\left(\left|x_{1}^{*}\left(v_{i}\right)\right|^{\tilde{y}}, \cdots, \mid x_{n}^{*}\left(v_{n}\right) \tilde{\mid}\right)+h^{*}(\tilde{\epsilon}, \cdots, \tilde{\epsilon}) \text {. }
\end{aligned}
$$

Thus,

$$
h^{*}\left(\left\|x_{1}^{*}\right\|^{\tilde{1}}, \cdots,\left\|x_{n}^{*}\right\| \tilde{)}\right) \leqq s+h^{*}(\tilde{\epsilon}, \cdots, \tilde{\epsilon})
$$

and since the continuity of $h^{*}$ implies that the last term can be made arbitrarily small, we have $s \geqq h^{*}\left(\left\|x_{1}^{*}\right\|^{\sim}, \cdots,\left\|x_{n}^{*}\right\|^{\sim}\right)$, and we obtain from (4a)

$$
\left\|\left(x_{1}^{*}, \cdots, x_{n}^{*}\right)\right\| \geqq h^{*}\left(\left\|x_{1}^{*}\right\| \tilde{}, \cdots,\left\|x_{n}^{*}\right\| \tilde{)}\right) \text {. }
$$

On the other hand, taking the following supremum over nonzero $\left(x_{1}, \cdots, x_{n}\right) \in X$ and noting that $\left\|\left(x_{1}, \cdots, x_{n}\right)\right\|=h\left(\left\|x_{1}\right\|, \cdots,\left\|x_{n}\right\|\right)$,

$$
\begin{aligned}
\left\|\left(x_{1}^{*}, \cdots, x_{n}^{*}\right)\right\| & =\sup \frac{\left|\sum x_{j}^{*}\left(x_{i}\right)\right|}{\left\|\left(x_{1}, \cdots, x_{n}\right)\right\|} \leqq \sup \frac{\sum\left\|x_{i}^{*}\right\|\left\|x_{j}\right\|}{\left\|\left(x_{1}, \cdots, x_{n}\right)\right\|} \\
& =\sup _{\alpha_{i} \geq 0} \frac{\sum \alpha_{i}\left\|x_{i}^{*}\right\|}{h\left(\alpha_{1}, \cdots, \alpha_{n}\right)} \leqq h^{*}\left(\left\|x_{1}^{*}\right\| \tilde{T}, \cdots,\left\|x_{n}^{*}\right\| \tilde{)}\right) .
\end{aligned}
$$

Combining this with (5), the theorem is proved.

Corollary 1. (a) For any element $\left(x_{1}^{*}, \cdots, x_{n}^{*}\right) \in X^{*}$,

$$
\left\|\left(x_{1}^{*}, \cdots, x_{n}^{*}\right)\right\|=\sup _{\alpha_{i} \geq 0} \frac{\sum_{i} \alpha_{i}\left\|x_{i}^{*}\right\|}{h\left(\alpha_{1}, \cdots, \alpha_{n}\right)},
$$

where $h$ is the norm on $C_{n}$ associated with the norm on $X$.

(b) For any $\left(x_{1}, \cdots, x_{n}\right) \in X$,

$$
\left\|\left(x_{1}, \cdots, x_{n}\right)\right\|=\sup _{\alpha_{i} \geq 0} \frac{\sum \alpha_{i}\left\|x_{i}\right\|}{h^{*}\left(\alpha_{1}, \cdots, \alpha_{n}\right)},
$$

where $h^{*}$ is the norm on $C_{n}^{*}$ associated with the norm on $X^{*}$.

Proof. (a) In the last steps of the proof of the theorem, we have equality at each stage and so the first result follows from (6).

(b) There is a norm-preserving isomorphism $\phi$ of $X$ onto a subset $\hat{X}$ of the second dual $X^{* *}$ (cf. Dunford and Schwartz [2] II.3.19) and, as above, we may identify $X^{* *}$ with $\sum_{i} \oplus X_{i}^{* *}$. Thus, to each $z=\left(x_{1}, \cdots, x_{n}\right) \in X$ corresponds $\varepsilon=\phi(z) \in X^{* *}$ and, for all $x^{*} \in X^{*}, \varepsilon\left(x^{*}\right)=x^{*}(z)$ or,

$$
\left(\hat{x}_{1}, \cdots, \hat{x}_{n}\right)\left(x_{1}^{*}, \cdots, x_{n}^{*}\right)=\left(x_{1}^{*}, \cdots, x_{n}^{*}\right)\left(x_{1}, \cdots, x_{n}\right) .
$$

Applying (a) to evaluate $\|\xi\|$, we have

$$
\|z\|=\|\hat{z}\|=\sup _{\alpha_{i} \geq 0} \frac{\sum \alpha_{i}\left\|\hat{x}_{i}\right\|}{h^{*}\left(\alpha_{1}, \cdots, \alpha_{n}\right)} .
$$

However, if $\phi_{i}$ is the norm-preserving isomorphism of $X_{i}$ onto $\hat{X}_{i} \subseteq X_{i}^{* *}$, then $\phi$ may be defined by

$$
\phi\left(x_{1}, \cdots, x_{n}\right)=\left(\phi_{1} x_{1}, \cdots, \phi_{n} x_{n}\right)=\left(\hat{x}_{1}, \cdots, \hat{x}_{n}\right) .
$$


Thus, if we identify $z$ with $z$, we may also identify $x_{i}$ with $\hat{x}_{i}$ for $j=1,2, \cdots, n$, and we obtain

$$
\|z\|=\sup _{\alpha_{i} \geq 0} \frac{\sum \alpha_{i}\left\|x_{i}\right\|}{h^{*}\left(\alpha_{1}, \cdots, \alpha_{n}\right)} .
$$

In the next corollary, we present another characterization of absolute norms in terms of a generalized Hölder inequality.

COROllary 2. A norm on $X$ is absolute iff

$$
\sum_{i}\left\|x_{i}\right\|\left\|x_{i}^{*}\right\| \leqq\|z\|\left\|z^{*}\right\|
$$

for all $z=\left(x_{1}, \cdots, x_{n}\right) \in X, z^{*}=\left(x_{1}^{*}, \cdots, x_{n}^{*}\right) \in X^{*}$.

Proof. For any norm on $X$, let $z=\left(x_{1}, \cdots, x_{n}\right) \in X$ and suppose that, under the natural embeddings, $z \rightarrow \hat{z} \in X^{* *}$ and $x_{i} \rightarrow \hat{x}_{i} \in X_{i}^{* *}$ for $j=1,2, \cdots, n$. Then,

$$
\|z\|=\|\hat{z}\|=\sup _{z^{*}} \frac{\left|\sum \hat{x}_{i}\left(x_{i}^{*}\right)\right|}{\left\|z^{*}\right\|} \leqq \sup _{z^{*}} \frac{\sum\left\|\hat{x}_{i}\right\|\left\|x_{i}^{*}\right\|}{\left\|z^{*}\right\|}=\sup _{\mathbf{s}^{*}} \frac{\sum\left\|x_{i}^{*}\right\|\left\|x_{i}\right\|}{\left\|z^{*}\right\|}
$$

However, the generalized Hölder inequality (7) implies $\|z\| \geqq \sum\left(\left\|x_{i}^{*}\right\|\left\|x_{i}\right\| /\left\|z^{*}\right\|\right)$ for each nonzero $z^{*} \in X^{*}$ and so

$$
\|z\|=\sup _{z^{*}} \frac{\sum\left\|x_{i}^{*}\right\|\left\|x_{i}\right\|}{\left\|z^{*}\right\|}
$$

and we see at once that the norm on $X$ is absolute.

Conversely, if we are given an absolute norm on $X$, then part (b) of Corollary 1 and Theorem 3 give

$$
\|z\|=\sup _{\alpha_{i} \geq 0} \frac{\sum \alpha_{i}\left\|x_{i}\right\|}{h^{*}\left(\alpha_{1}, \cdots, \alpha_{n}\right)}=\sup _{z^{*}} \frac{\sum\left\|x_{i}^{*}\right\|\left\|x_{i}\right\|}{\left\|z^{*}\right\|}
$$

and the inequality (7) follows.

4. Norms on Tensor Products. We now confine our attention to finite-dimensional normed linear spaces $X$ and $Y$ and consider the construction of norms on the tensor product $X \otimes Y$. If $E=\left\{e_{1}, e_{2}, \cdots, e_{m}\right\}$ and $F=\left\{f_{1}, f_{2}, \cdots, f_{n}\right\}$ are bases for $X, Y$, respectively, then $\left\{e_{i} \otimes f_{k}: 1 \leqq j \leqq m, 1 \leqq k \leqq n\right\}$ is a basis for $X \otimes Y$. Furthermore, every element $z$ of $X \otimes Y$ has a unique representation in the form $z=\sum_{i} e_{i} \otimes y_{i}$ where $y_{1}, \cdots, y_{m} \in Y$ and, similarly, in the form $z=\sum_{k} x_{k} \otimes f_{k}$. An element of $X \otimes Y$ is decomposable if it is expressible in the form $x \otimes y$ where $x \in X, y \in Y$. By means of the isomorphism $\lambda \otimes x \leftrightarrow \lambda x$, we shall subsequently identify $C \otimes X$ with $X$.

If a norm on $X \otimes Y$ has the property $\|x \otimes y\|=\|x\|\|y\|$ for all decomposable elements of $X \otimes Y$, it is called a crossnorm. Such norms (and operator norms, in particular) are of special interest. The prime example is the absolute value norm on the complex numbers.

Now let $x^{*} \in X^{*}, y^{*} \in Y^{*}$, then $x^{*} \otimes y^{*} \in X^{*} \otimes Y^{*}$ but may also be interpreted as a linear functional on $X \otimes Y$ which is characterized by

$$
\left(x^{*} \otimes y^{*}\right)(x \otimes y)=x^{*}(x) y^{*}(y) \text {. }
$$

Let $E^{*}=\left\{e_{1}^{*}, \cdots, e_{m}^{*}\right\}$ and $F^{*}=\left\{f_{1}^{*}, \cdots, f_{n}^{*}\right\}$ be dual bases for $E$ and $F$, respectively, 
so that $e_{i}^{*}\left(e_{j}\right)=\delta_{i j}, 1 \leqq i, j \leqq m$ and $f_{k}^{*}\left(f_{l}\right)=\delta_{k l}, 1 \leqq k, l \leqq n$. Then, $\left\{e_{i}^{*} \otimes f_{k}^{*}\right.$ : $1 \leqq j \leqq m, 1 \leqq k \leqq n\}$ is a basis for $X^{*} \otimes Y^{*}$. But this is also the dual of the basis $\left\{e_{i} \otimes f_{k}\right\}$ for $X \otimes Y$ for, by (8),

$$
\left(e_{i}^{*} \otimes f_{k}^{*}\right)\left(e_{i} \otimes f_{l}\right)=e_{i}^{*}\left(e_{i}\right) f_{k}^{*}\left(f_{l}\right)=\delta_{i j} \delta_{k l}=\delta_{(i, k),(i, l)} .
$$

Thus, functionals of the form $x^{*} \otimes y^{*} \operatorname{span}(X \otimes Y)^{*}$ which may therefore be identified with $X^{*} \otimes Y^{*}$ and, without ambiguity, $x^{*} \otimes y^{*}$ may represent either the tensor product of functionals $x^{*} \in X^{*}$ and $y^{*} \in Y^{*}$, or a decomposable element of $X^{*} \otimes Y^{*}$.

If $z^{*}=\sum_{i} e_{i}^{*} \otimes y_{i}^{*} \in X^{*} \otimes Y^{*}$ and $z=\sum_{i} e_{i} \otimes y_{i}$ is a typical member of $X \otimes Y$, we may write

$$
\left\|z^{*}\right\|=\sup _{z} \frac{\left|z^{*} z\right|}{\|z\|}=\sup \frac{\left|\sum e_{i}^{*} \otimes y_{i}^{*} \sum e_{i} \otimes y_{i}\right|}{\|z\|}=\sup \frac{\left|\sum y_{i}^{*} y_{i}\right|}{\|z\|} .
$$

If $X, Y, U, V$ are linear spaces and $A, B$ are linear operators $A: X \rightarrow U$ and $B: Y \rightarrow V$, then the tensor product $A \otimes B$ may be defined as a linear operator from $X \otimes Y$ into $U \otimes V$ by

$$
(A \otimes B) \sum_{i} e_{i} \otimes y_{i}=\sum_{i}\left(A e_{i}\right) \otimes\left(B y_{i}\right)
$$

Lemma. If $U, V, X, Y$ are normed linear spaces, $A: X \rightarrow U$ and $B: Y \rightarrow V$ are linear operators, and if the norms on $X \otimes Y$ and $U \otimes V$ are crossnorms, then $\|A \otimes B\| \geqq\|A\|\|B\|$.

Proof. Let $D$ be the set of nonzero decomposable elements of $X \otimes Y$ and note first that, if $x \otimes y \in D$,

$$
\|(A \otimes B)(x \otimes y)\|=\|(A x) \otimes(B y)\|=\|A x\|\|B y\|,
$$

since the norm on $U \otimes V$ is also a crossnorm. Then, since the norm on $X \otimes Y$ is also a crossnorm,

$$
\begin{aligned}
\|A \otimes B\| & =\sup _{v \in X \otimes Y} \frac{\|(A \otimes B) v\|}{\|v\|} \geqq \sup _{v \in D} \frac{\|(A \otimes B) v\|}{\|v\|} \\
& =\sup _{x \otimes v \in D} \frac{\|A x\|}{\|x\|} \cdot \frac{\|B y\|}{\|y\|}=\|A\|\|B\| .
\end{aligned}
$$

In the case that $U=V=C$, we deduce that, if the norm on $X \otimes Y$ is a crossnorm, then $\left\|x^{*} \otimes y^{*}\right\| \geqq\left\|x^{*}\right\|\left\|y^{*}\right\|$. Schatten [4] demonstrates a class of crossnorms on $X \otimes Y$ for which the strict inequality generally obtains in the dual spaces. We shall show how the concept of "absolute" norms can be used in this situation to obtain equality for the functionals of the dual spaces and, also, for the case $U=X$, $V=Y$.

We shall say that a norm on (a) $X$, or (b) $X \otimes Y$ is $E$-absolute if

(a) $\left|\xi_{j}\right|=\left|\eta_{j}\right|, j=1,2, \cdots, m$, implies $\left\|\sum_{i} \xi_{i} e_{i}\right\|=\left\|\sum \eta_{j} e_{i}\right\|$, or

(b) $\left\|y_{i}\right\|=\left\|a_{i}\right\|, j=1,2, \cdots, m$, and $y_{i}, a_{i} \in Y$ imply $\left\|\sum e_{i} \otimes y,\right\|$ $=\left\|\sum e_{i} \otimes a_{i}\right\|$, respectively.

Since the spaces $X$ and $X \otimes Y$ are isomorphic to direct sums

$$
C e_{1} \oplus \cdots \oplus C e_{m} \quad \text { and } \quad\left(e_{1} \otimes Y\right) \oplus \cdots \oplus\left(e_{m} \otimes Y\right)
$$


respectively, we may deduce from Theorem 1 corresponding monotonicity properties for $E$-absolute norms. We note also that, by Theorem 3 , once the basis $E$ is fixed, $E$-absolute norms (in either sense) correspond to absolute norms on $C_{n}$.

LEMMA. Let $X$ be a normed linear space, let the norm on $X$ be E-absolute and let $E^{*}=\left\{e_{1}^{*}, \cdots, e_{m}^{*}\right\} \subset X^{*}$ be the coordinate functions determined by $E$. Then, the (induced) norm on $X^{*}$ is $E^{*}$-absolute.

Proof. Using the first of the decompositions (9), the lemma is a consequence of Theorem 4.

THEOREM 5. Let $X, Y$ be normed linear spaces with a basis $E=\left\{e_{1}, \cdots, e_{m}\right\}$ for $X$ and let a crossnorm be defined on $X \otimes Y$. If the norm on $X \otimes Y$ is $E$-absolute, then

(a) the norm on $X$ is E-absolute,

(b) $\left\|\sum e_{i} \otimes y_{i}\right\|=\left\|\sum\right\| y_{i}\left\|e_{i}\right\|$, and

(c) $\left\|x^{*} \otimes y^{*}\right\|=\left\|x^{*}\right\|\left\|y^{*}\right\|$. That is, the norm on $X^{*} \otimes Y^{*}$ is also a crossnorm.

Proof. (a) Choose $v \in Y$ such that $\|v\|=1$. Then, if $x \in X$, we have

$$
\|x \otimes v\|=\|x\|\|v\|=\|x\| \text {. }
$$

Let $x=\sum_{i} \xi_{i} e_{j}$; then $\|x\|=\|x \otimes v\|=\left\|\sum e_{i} \otimes \xi_{i} v\right\|$ and, since the norm on $X \otimes Y$ is $E$-absolute,

$$
\|x\|=\left\|\sum e_{i} \otimes\left|\xi_{i}\right| v\right\|=\left\|\sum\left|\xi_{i}\right| e_{i} \otimes v\right\|=\left\|\sum\left|\xi_{i}\right| e_{i}\right\| .
$$

(b) We again choose $v \in Y$ with $\|v\|=1$ and, since the norm on $X \otimes Y$ is absolute, we have

$\left\|\sum e_{i} \otimes y_{i}\right\|=\left\|\sum e_{i} \otimes\right\| y_{i}\|v\|=\left\|\sum\right\| y_{i}\left\|e_{i} \otimes v\right\|=\left\|\sum\right\| y_{i}\left\|e_{i}\right\|$.

(c) If $x^{*}=\sum \xi_{i} e_{i}^{*} \in X^{*}$ then, by the lemma, the norm on $X^{*}$ is $E^{*}$-absolute and, by part (b),

$$
\left\|x^{*} \otimes y^{*}\right\|=\left\|\sum e_{i}^{*} \otimes \xi_{i} y^{*}\right\|=\left\|\sum\right\| \xi_{i} y^{*}\left\|e_{i}^{*}\right\| .
$$

Thus, using part (a),

$$
\left\|x^{*} \otimes y^{*}\right\|=\left\|y^{*}\right\|\left\|\sum\left|\xi_{i}\right| e_{i}^{*}\right\|=\left\|y^{*}\right\|\left\|\sum \xi_{i} e_{i}^{*}\right\|=\left\|x^{*}\right\|\left\|y^{*}\right\| \text {. }
$$

These results suggest that the formula of part (b) may provide a useful class of norms of $X \otimes Y$. We have

THEOREM 6. Let $X, Y$ be normed linear spaces, let $E=\left\{e_{1}, \cdots, e_{m}\right\}$ be a basis for $X$ and let the norm on $X$ be E-absolute. Then, the function $\|\cdot\|_{1}$ defined on $X \otimes Y$ by

$$
\left\|\sum e_{i} \otimes y_{i}\right\|_{1}=\left\|\sum\right\| y_{i}\left\|e_{i}\right\|
$$

is a norm on $X \otimes Y$ which is $E$-absolute and is a crossnorm.

Proof. Using the isomorphisms (9), we may identify $X$ with $C_{n}$ and $X \otimes Y$ with $Y_{m}(=Y \oplus \cdots \oplus Y)$ as follows:

$$
\sum_{i=1}^{m} \xi_{i} e_{i} \leftrightarrow\left(\xi_{1}, \cdots, \xi_{m}\right) \text { and } \sum_{i=1}^{m} e_{i} \otimes y_{i} \leftrightarrow\left(y_{1}, \cdots, y_{m}\right) .
$$

By Theorem 3, the $E$-absolute norm on $X$ determines an underlying absolute norm $h$ on $C_{n}$ for which $\left\|\sum\right\| y_{j}\left\|e_{i}\right\|=h\left(\left\|y_{1}\right\|, \cdots,\left\|y_{m}\right\|\right)$ and, in its turn, $h$ determines an absolute norm on $X \otimes Y$ for which 


$$
\left\|\sum e_{i} \otimes y_{i}\right\|=h\left(\left\|y_{1}\right\|, \cdots,\left\|y_{m}\right\|\right)=\left\|\sum\right\| y_{i}\left\|e_{i}\right\| .
$$

But this norm is just the function $\|\cdot\|_{1}$ and so we obtain the first part of the theorem. To see that the resulting norm is a crossnorm, let $y \in Y$ and $x=\sum \xi_{i} e_{i} \in X$. Then, $\|x \otimes y\|_{1}=\left\|\sum e_{i} \otimes \xi_{i} y\right\|_{1}=\left\|\sum\right\| \xi_{i} y\left\|e_{i}\right\|=\left\|\sum\left|\xi_{i}\right| e_{i}\right\|\|y\|=\|x\|\|y\|$, and the theorem is proved.

We note that, by Theorem 5 , the norm induced in $X^{*} \otimes Y^{*}$ by $\|\cdot\|_{1}$ is also a crossnorm.

Corollary. Let $X, Y$ be normed linear spaces. Let $F=\left\{f_{1}, \cdots, f_{n}\right\}$ be a basis for $Y$ and let the norm on $Y$ be F-absolute. Then, the function $\|\cdot\|_{2}$ defined on $X \otimes Y$ by

$$
\left\|\sum_{k} x_{k} \otimes f_{k}\right\|_{2}=\left\|\sum_{k}\right\| x_{k}\left\|f_{k}\right\|
$$

defines a norm on $X \otimes Y$ which is $F$-absolute and is a crossnorm.

The proof is the obvious parallel of that for Theorem 6 .

5. Norms of Tensor Products of Operators. We now consider the definition of operator norms. If $X$ is a linear space and $L(X)$ is the linear space of bounded linear operators from $X$ into itself, then we require the usual vector norm axioms for a norm on $L(X)$ together with the submultiplicative property: $\|A B\| \leqq\|A\|\|B\|$ for all $A, B \in L(X)$. Then, $L(X)$ is a normed algebra.

If $X, Y$ are finite-dimensional linear spaces, we now are interested in the tensor product $L(X) \otimes L(Y)$. This is not only a linear space; it is an algebra in which

$$
\left(A_{1} \otimes B_{1}\right)\left(A_{2} \otimes B_{2}\right)=A_{1} A_{2} \otimes B_{1} B_{2}
$$

holds for all $A_{1}, A_{2} \in L(X)$ and $B_{1}, B_{2} \in L(Y)$. As such, $L(X) \otimes L(Y)$ may be identified with the algebra $L(X \otimes Y)$ in such a way that the element $A \otimes B$ of $L(X) \otimes L(Y)$ is identified with the "tensor product" $A \otimes B$ of the operators $A, B$.

If $L(X), L(Y)$ are finite-dimensional normed algebras, we are to use the norms on $L(X), L(Y)$ to define a norm on $L(X \otimes Y)$ which is submultiplicative and will be a crossnorm. That is, if $M, N \in L(X \otimes Y)$, then $\|M N\| \leqq\|M\|\|N\|$ and if $A \in L(X), B \in L(Y),\|A \otimes B\|=\|A\|\|B\|$.

The first suggestion is to apply Theorem 6 directly after picking out a basis for $L(X)$. The results of Theorem 6 then guarantee all the required properties of the norm on $L(X \otimes Y)$ with the exception of the submultiplicative property. That is, $L(X \otimes Y)$ need not be a normed algebra. In the following case, we have the submultiplicative property.

Let $X$ be the space $C_{m \times m}$ of $m \times m$ complex matrices and let $E_{i j} \in C_{m \times m}$ be the matrix with a one in the $i, j$ position and zeros elsewhere. Then, $E=\left\{E_{i i}: 1 \leqq i\right.$, $j \leqq m\}$ is a basis for $C_{m \times m}$. Our result applies to $m \times m$ matrices whose elements belong to a normed linear algebra, an algebra of bounded linear operators, for example.

THEOREM 7. Let $Y$ be a normed linear algebra and suppose a norm is given on $C_{m \times m}$ which is submultiplicative. If $B \in C_{m \times m} \otimes Y$ let $B=\sum_{1 \leq i, i \leq m} E_{i j} \otimes B_{i i}$ and suppose further that the norm on $C_{m \times m}$ is $E$-absolute. Then, the function $\|\cdot\|_{1}$ defined on $C_{m \times m} \otimes Y b y$ 


$$
\|B\|_{1}=\left\|\sum_{i, j}\right\| B_{i i}\left\|E_{i u}\right\|
$$

is a submultiplicative norm and is a crossnorm.

Proof. As noted above, we have only to prove the submultiplicative property. Let $A, B \in C_{m \times m} \otimes Y$ with

$$
A=\sum_{i, j} E_{i j} \otimes A_{i i}, \quad B=\sum_{i, j} E_{i j} \otimes B_{i j} .
$$

Then, since $E_{i k} E_{l j}=\delta_{k l} E_{i j}$, we obtain from (10) and the usual matrix multiplication

$$
A B=\left(\sum E_{i k} \otimes A_{i k}\right)\left(\sum E_{l i} \otimes B_{l i}\right)=\sum_{i, j}\left(E_{i j} \otimes \sum_{k} A_{i k} B_{k i}\right),
$$

and

$$
\|A B\|_{1}=\left\|\sum_{i, i}\right\| \sum_{k} A_{i k} B_{k i}\left\|E_{i i}\right\| .
$$

Now, the norm on $C_{m \times m}$ is monotonic (with respect to $E$ ) and the norm on $Y$ is submultiplicative, so

$$
\begin{aligned}
\|A B\|_{1} & \leqq\left\|\sum_{i, i}\left(\sum_{k}\left\|A_{i k}\right\|\left\|B_{k i}\right\|\right) E_{i j}\right\| \\
& =\left\|\sum_{i, j}\left(\sum_{k}\left\|A_{i k}\right\| E_{i k}\right)\left(\sum_{l}\left\|B_{l i}\right\| E_{l i}\right)\right\| \\
& =\left\|\left(\sum_{i, k}\left\|A_{i k}\right\| E_{i k}\right)\left(\sum_{i, l}\left\|B_{i j}\right\| E_{l i}\right)\right\| .
\end{aligned}
$$

But then the norm on $C_{m \times m}$ is submultiplicative so that

$$
\|A B\|_{1} \leqq\left\|\sum_{i, k}\right\| A_{i k}\left\|E_{i k}\right\| \cdot\left\|\sum_{i, l}\right\| B_{i j}\left\|E_{l i}\right\|=\|A\|_{1}\|B\|_{1} .
$$

We remark that, with the norm of this theorem, $\|A\|_{1}$ is equated to the norm (in $C_{m \times m}$ ) of the nonnegative matrix [ $\left.\left\|A_{i j}\right\|\right]$ which, by the classical Perron-Frobenius theorem, has a maximum nonnegative eigenvalue $\lambda$. If $Y$ is an algebra of bounded linear operators so that $A_{i i} \in L(S)$ for some linear space $S$, then $A \in L\left(S_{n}\right)$ and the proof of a theorem of Ostrowski (Theorem 4 of [3]) can be used to show that the eigenvalues of $A$ (if any) cannot exceed $\lambda$ in absolute value.

We now turn our attention to the formulation of operator (bound) norms in the usual way from the norms on the underlying spaces. Thus, if $A: X \rightarrow X,\|A\|=$ $\sup _{x \in X}\|A x\| /\|x\|$.

We note first that if $A: X \rightarrow X$ and $B: Y \rightarrow Y$ are linear operators and if the norm on $X \otimes Y$ is a crossnorm, then by the first lemma of $\S 4,\|A \otimes B\| \geqq\|A\|,\|B\|$. Once again, we are interested in those norms for which equality obtains.

Consider the norms $\|\cdot\|_{1}$ and $\|\cdot\|_{2}$ defined on $X \otimes Y$ in Theorem 6 and its Corollary. We shall use the same subscripts for the norm defined on $L(X \otimes Y)$ by these vector norms. We denote the identity mappings on $X$ and $Y$ by $I_{X}, I_{Y}$ respectively.

Lemma. (i) Let the norm on $X$ be E-absolute and $B \in L(Y)$, then

$$
\left\|I_{X} \otimes B\right\|_{1}=\|B\| .
$$

(ii) Let the norm on $Y$ be F-absolute and $A \in L(X)$, then 


$$
\left\|A \otimes I_{Y}\right\|_{2}=\|A\| \text {. }
$$

Proof. We shall only prove (i). Part (ii) is proved by a similar argument. Writing $z=\sum e_{i} \otimes y_{i}$ for a typical element of $X \otimes Y$,

$$
\left\|I_{X} \otimes B\right\|_{1}=\sup _{z} \frac{\left\|\sum e_{i} \otimes B_{y_{i}}\right\|}{\left\|\sum e_{i} \otimes y_{j}\right\|}=\sup _{y_{j} \in Y} \frac{\left\|\sum\right\| B_{y_{j}}\left\|e_{i}\right\|}{\left\|\sum\right\| y_{i}\left\|e_{i}\right\|}
$$

and using the monotonic property of the norm on $X$, we obtain $\left\|I_{X} \otimes B\right\|_{1} \leqq\|B\|$. However, we have noted that, for a crossnorm on $X \otimes Y,\left\|I_{X} \otimes B\right\| \geqq\left\|I_{X}\right\|\|B\|=$ $\|B\|$. Part (i) is obtained.

THEOREM 8. Let $X, Y$ be finite-dimensional normed linear spaces with bases $E$ and $F$, respectively. If the norms on $X, Y$ are $E$-absolute and $F$-absolute, respectively, and if the vector norms $\|\cdot\|_{1}$ and $\|\cdot\|_{2}$ of Theorem 6 coincide, then $\|A \otimes B\|=\|A\|\|B\|$ for the corresponding induced norms.

Proof. We observe that $A \otimes B=\left(A \otimes I_{Y}\right)\left(I_{X} \otimes B\right)$ and, since an induced norm is necessarily submultiplicative,

$$
\|A \otimes B\| \leqq\left\|A \otimes I_{Y}\right\|\left\|I_{X} \otimes B\right\| .
$$

The lemma then gives $\|A \otimes B\| \leqq\|A\|\|B\|$ and, since we have already proved $\|A \otimes B\| \geqq\|A\|\|B\|$, the theorem is proved.

We note that it is always the case that $\|x \otimes y\|_{1}=\|x \otimes y\|_{2}$, since both norms are crossnorms. In the theorem, we suppose this true for all elements of $X \otimes Y$ and not just the decomposable elements.

6. Application to Vectors and Matrices. Let $X, Y$ be linear spaces with $E=$ $\left\{e_{1}, \cdots, e_{m}\right\}$ a basis for $X$ and $F=\left\{f_{1}, \cdots, f_{n}\right\}$ a basis for $Y$. Then, linear operators $A: X \rightarrow X$ and $B: Y \rightarrow Y$ have matrix representations $A_{M} \in C_{m \times m}$, with respect to $E$, and $B_{M} \in C_{n \times n}$ with respect to $F$. We may choose as a basis for $X \otimes Y$ the vectors $e_{1} \otimes f_{1}, e_{1} \otimes f_{2}, \cdots, e_{1} \otimes f_{n}, e_{2} \otimes f_{1}, \cdots, e_{2} \otimes f_{n}, \cdots, e_{m} \otimes f_{1}, \cdots, e_{m} \otimes f_{n}$,

in this order, and it is easily seen that the matrix representation of $A \otimes B$ with respect to this basis is the familiar Kronecker, or direct product of the matrices $A_{M}, B_{M}$, written $A_{M} \otimes B_{M}$.

The unit vectors $e_{k}$ in the space $C_{m}^{\prime}$ of column vectors have a one in the $k$ th place and zeros elsewhere. In the case $X=C_{m}^{\prime}, Y=C_{n}^{\prime}$, we may choose bases $E$ and $F$ of unit vectors and then the above basis for $C_{m}^{\prime} \otimes C_{n}^{\prime}=C_{m n}^{\prime}$ is also of unit vectors. The norm of $C_{m}^{\prime}$ is $E$-absolute if, for all pairs $x, y \in C_{m}^{\prime}$ with $\left|x_{i}\right|=\left|y_{i}\right|$ for $j=1$, $2, \cdots, m$, we have $\|x\|=\|y\|$. This now coincides with an absolute vector norm in the usual matrix theoretic sense (Bauer, et al. [1]).

Let $a \in C_{m}^{\prime} \otimes C_{n}^{\prime}$. Then, there are complex numbers $\lambda_{i k}$ for which

$$
a=\sum_{k=1}^{n} \sum_{i=1}^{m} \lambda_{i k}\left(e_{i} \otimes f_{k}\right)
$$

and we may also write

$$
a=\sum_{i=1}^{m_{1}} e_{i} \otimes y^{(j)}=\sum_{k=1}^{n} x^{(k)} \otimes f_{k},
$$


where

$$
y^{(i)}=\sum_{k} \lambda_{j k} f_{k}=\left[\begin{array}{c}
\lambda_{j 1} \\
\lambda_{i 2} \\
\vdots \\
\lambda_{i n}
\end{array}\right], \quad x^{(k)}=\sum_{i} \lambda_{j k} e_{i}=\left[\begin{array}{c}
\lambda_{1 k} \\
\lambda_{2 k} \\
\vdots \\
\lambda_{n k}
\end{array}\right] .
$$

In the norms of Theorem 6 and its Corollary, we see that $\|a\|_{1}$ and $\|a\|_{2}$ are the $X$-norm (norm in $C_{m}^{\prime}$ ) and $Y$-norm (norm in $C_{n}^{\prime}$ ), respectively, of

$$
\left[\begin{array}{c}
\left\|y^{(1)}\right\| \\
\left\|y^{(2)}\right\| \\
\vdots \\
\left\|y^{(m)}\right\|
\end{array}\right] \text { and }\left[\begin{array}{c}
\left\|x^{(1)}\right\| \\
\left\|x^{(2)}\right\| \\
\vdots \\
\left\|x^{(n)}\right\|
\end{array}\right]
$$

In particular, if $d=x \otimes y$ is a decomposable member of $C_{m}^{\prime} \otimes C_{n}^{\prime}$, then $\|d\|_{1}=$ $\|d\|_{2}=\|x\|\|y\|$, since both norms on $C_{m}^{\prime} \otimes C_{n}^{\prime}$ are crossnorms (Theorem 6).

As an application of Theorem 7, we take for the space $Y$ the $n \times n$ complex matrices with an appropriate norm and the operator $A$ is then an $m n \times m n$ partitioned matrix. The norm on $m n \times m n$ matrices is then constructed from the norms of the $n \times n$ blocks as indicated and, provided the norm on $C_{m \times m}$ depends only on the absolute values of matrix elements, the resulting norm on $C_{m n \times m n}$ is a crossnorm. A very special example is the case of a $p$-norm $(1 \leqq p \leqq 2)$ used in both spaces $C_{m \times m}$, $C_{n \times n}$ which yields the same $p$-norm in $C_{m n \times m n}$. That is, for a matrix $A \in C_{m \times m}$, for example,

$$
\|A\|=\left(\sum_{i, i=1}^{m}\left|a_{i i}\right|^{p}\right)^{1 / p}
$$

It is a trivial matter to check the crossnorm property directly in this case.

To illustrate Theorem 8 , suppose that $C_{m}^{\prime}, C_{n}^{\prime}$ have the same $p$-norm imposed on them. Since these are merely vector norms, we may have $1 \leqq p \leqq \infty$ in this case. Then, $\|\cdot\|_{1}$ and $\|\cdot\|_{2}$ coincide and yield the same $p$-norm on $C_{m n}^{\prime}=C_{m}^{\prime} \otimes C_{n}^{\prime}$. The operator norms in Theorem 8 are then those induced by the vector $p$-norms and are again crossnorms.

It is noteworthy that, for these norms and for $1 \leqq p \leqq 2$, Theorem 7 is not included in Theorem 6 . To see this, we have only to show that a matrix norm induced by an absolute vector norm is not necessarily absolute (with respect to the basis $\left\{E_{i j}\right\}$ in $C_{m \times m}$ ). Consider the case $p=2$ (the euclidean vector norm) and the matrices

$$
A=\left[\begin{array}{ll}
1 & 1 \\
1 & 1
\end{array}\right], \quad B=\left[\begin{array}{rr}
1 & -1 \\
1 & 1
\end{array}\right] .
$$

It is well known that the matrix norm induced by this vector norm is the spectral norm and, for any matrix $A$, is given by the square root of the largest eigenvalue of $A^{*} A$ (star denotes a conjugate transpose). The norms of matrices $A$ and $B$ with respect to an $\left\{E_{i j}\right\}$-absolute norm are obviously equal. However, their spectral norms are 2 and $\sqrt{ } 2$, respectively. 
This case may be contrasted with the cases of $p=1$ and $p=\infty$ for the vector norm. It is well known that the induced matrix norms are $\left\{E_{i i}\right\}$-absolute in these two cases.

Department of Mathematics, Statistics and Computing Science The University of Calgary

Calgary, Alberta, Canada

1. F. L. Bauer, J. Stoer \& C. Witzgall, "Absolute and monotonic norms," Numer. Math., v. 3, 1961, pp. 257-264. MR 23 \#B3136.

2. N. DUNFORD \& J. T. SCHWARTZ, Linear Operators. I: General Theory, Pure and Appl. Math., vol. 7, Interscience, New York, 1958. MR 22 \#8302.

3. A. M. OsTROWSKI, "On some metrical properties of operator matrices and matrices partitioned into blocks," J. Math. Anal. Appl., v. 2, 1961, pp. 161-209. MR 24 \#A421.

4. R. Schatten, A Theory of Cross-Spaces, Ann. of Math. Studies, no. 26, Princeton Univ. Press, Princeton, N. J., 1950. MR 12, 186. 